\section{Primer puente a trasplante cardiaco exitoso en Chile usando el dispositivo Heart Mate II}

\author{
NICOLÁS BUNSTER ${ }^{1}$, MAURICIO VILLAVICENCIO ${ }^{1,2}$, JONGSUNG LIM ${ }^{1}$, \\ ERIKA DONOSO ${ }^{1}$, FRANCESCA GAJARDO $^{1}$, VÍCTOR ROSSEL ${ }^{1,3}$
}

\section{First successful bridge to cardiac transplantation in Chile using the Heart Mate II device. Report of one case}

Implantable ventricular assist devices are an effective treatment option for end-stage heart failure patients as a bridge to cardiac transplantation, to improve the clinical condition and organ function allowing discharge from the hospital to await for transplantation. The second alternative is to use the device as destination therapy for patients with contraindications for cardiac transplantation, in whom it is maintained indefinitely. We report a 43-year-old patient, with a dilated cardiomyopathy, severe left ventricular dysfunction and advanced heart failure. A ventricular assist device Heart Mate $I^{\circ}{ }^{\circ}$, as a bridge to transplantation, was implanted to the patient in the United States. It was explanted for the first time in Chile at the National Thorax Institute. Heart transplantation was performed using the bicaval technique. Induction of immunosuppression was done with basiliximab. Generic immunosuppression was carried out with cyclosporine, mycophenolate mofetil and prednisone. Postoperatively the patient evolved with right femoral vein thrombosis in the femoral cannulation site, phlegmasia alba dolens, rhabdomyolysis, oliguric acute renal failure, which required renal replacement therapy, severe shock, with high requirements of vasoactive drugs and need for mechanical ventilation. He required a reoperation for hemothorax and had an Enterobacter pneumonia. After a period of serious illness, he began a gradual recovery and was discharged from the hospital after 58 days. After two years, he remains in functional class $I$, with a normal graft function.

(Rev Med Chile 2014; 142: 521-525)

Key words: Chile; Heart assist devices; Heart transplantation.

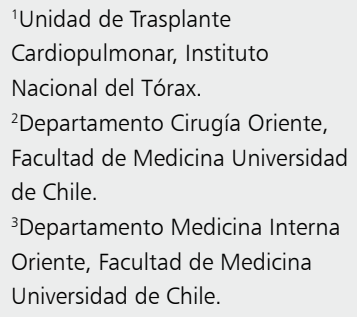

Recibido el 16 de septiembre de 2013, aceptado el 18 de marzo de 2014.

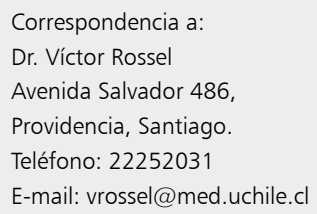

I a insuficiencia cardiaca (IC) es un importante problema de salud pública. Según datos de la American Heart Association se diagnostican 550.000 casos nuevos cada año, de los cuales 250.000 corresponden a cuadros de IC avanzada a pesar de un tratamiento adecuado ${ }^{1}$, con cifras de mortalidad que alcanzan hasta $33,3 \%$ al año ${ }^{2}$, generando costos muy altos ${ }^{3}$.

El tratamiento médico, el resincronizador y el desfibrilador automático implantable (DAI) han logrado mejorar la sobrevida y la calidad de vida de pacientes con $\mathrm{IC}^{4}$, sin embargo, en un número significativo de ellos, la enfermedad continúa avanzando y pasa al estadio $\mathrm{D}^{5}$.

El trasplante cardiaco ( $\mathrm{TxC}$ ) mejora en forma significativa las expectativas de supervivencia en pacientes con IC terminal, alcanzando cifras de sobrevida de $85 \%$ al año y $70 \%$ a los 5 años ${ }^{6}$. Sin embargo, el gran problema es el número limitado de donantes. En Estados Unidos de Norteamérica (EU) cerca de 20.000 pacientes se beneficiarían de un TxC, pero sólo se logran realizar alrededor 
de 2.300 cada año, falleciendo hasta $16 \%$ de los pacientes enlistados ${ }^{7}$.

En este complejo escenario, los dispositivos de asistencia ventricular (VADs) aparecen como una opción válida, para mejorar la condición de un paciente previo al TxC (puente al trasplante) o como una terapia definitiva para aquéllos pacientes con contraindicaciones para TxC (terapia de destinación $)^{8,9}$.

Heart Mate II ${ }^{\circledR}$ (HM II) es un dispositivo de asistencia ventricular izquierda implantable, de flujo continuo, diseñado para uso prolongado, que ha mostrado buenos resultados en términos de sobrevida ${ }^{10,11}$. En nuestro país se han utilizado VADs de corta duración como puente a trasplante $^{12,13}$, pero no existe experiencia con este tipo de dispositivos de larga duración dado su elevado costo. Presentamos el primer explante realizado en nuestro país de un dispositivo HM II utilizado como puente a TxC.

\section{Caso clínico}

Paciente de 43 años con antecedentes de IC diagnosticada el año 2005, secundaria a miocardiopatía dilatada, con disfunción sistólica severa, fracción de eyección de ventrículo izquierdo (FEVI) 22\%, con implante de DAI en el año 2006. Se trasladó a EU, manteniendo control y tratamiento regular de su enfermedad con fosinopril $5 \mathrm{mg}$, metoprolol $50 \mathrm{mg}$, espironolactona $25 \mathrm{mg}$, digoxina $0,125 \mathrm{mg}$ y ácido acetilsalicílico $100 \mathrm{mg}$. Presentó varias hospitalizaciones por descompensación de IC.

En mayo de 2010 ingresó al Hospital Mount Sinai de Nueva York por nuevo episodio de descompensación que requirió apoyo inotrópico con dobutamina y milrinona en dosis altas. Seis días más tarde presentó paro cardiorrespiratorio en fibrilación ventricular, se reanimó y conectó a ventilación mecánica (VM), evolucionó con arritmias ventriculares refractarias por lo que se decidió implante de urgencia del HM II y anuloplastía tricuspidea (Dispositivo in situ, Figura 1).

Con posterioridad al implante del dispositivo presentó IC derecha, con respuesta adecuada a furosemida y milrinona endovenosa, con regresión progresiva de los síntomas, siendo dado de alta 20 días post implante.

Luego de 5 meses reingresó nuevamente por descargas frecuentes del DAI, secundario a taquicardias ventriculares y fibrilación auricular con respuesta ventricular rápida, lo que se atribuyó al contacto de la cánula de entrada del dispositivo con el septum. Se realizó ajuste de parámetros de HM II y se inició tratamiento con amiodarona. No volvió a presentar arritmias significativas. Antes del alta se realizó ecocardiograma que mostró: ventrículo izquierdo (VI) de tamaño normal, con FEVI 40\%, disfunción severa de ventrículo derecho (VD), con mínima insuficiencia tricuspidea.

En los controles posteriores, reapareció IC de predominio derecho, con progresivo deterioro de su capacidad funcional. Se trasladó a Chile al Instituto Nacional del Tórax (INT) para ingresar a lista de espera de TxC en nuestro país en mayo de 2011. Llegó en condición clínica estable, pero con disfunción hepática y renal, recibiendo tratamiento con fosinopril, furosemida, espironolactona, metoprolol, digoxina, ácido acetilsalicílico y warfarina. Se enlistó para TxC, status IA.

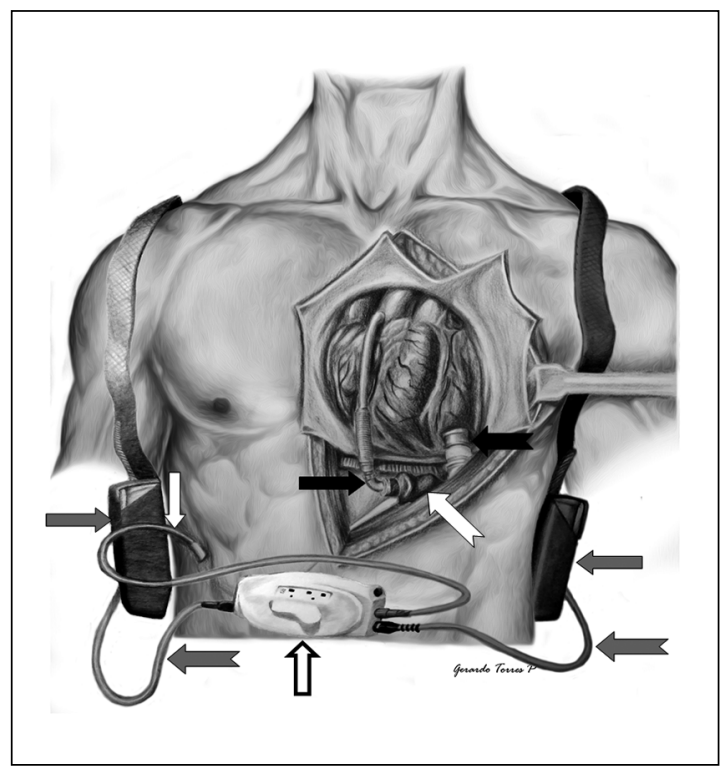

Figura 1. Esquema del dispositivo de asistencia ventricular izquierda Heart Mate II in situ, con los diferentes componentes del sistema. Cánula de entrada en el ápex del ventrículo izquierdo (flecha con muesca negra), cánula de salida hacia la raíz de la aorta (flecha negra), bomba de flujo axial rotatoria (flecha con muesca blanca), cable de conexión al controlador del dispositivo (flecha blanca), controlador del dispositivo, (flecha blanca con borde negro) cables de conexión a fuentes de energía, (flechas con muesca gris), batería (flecha gris) (cortesía de Enfermero Universitario Gerardo Cortés P.). 


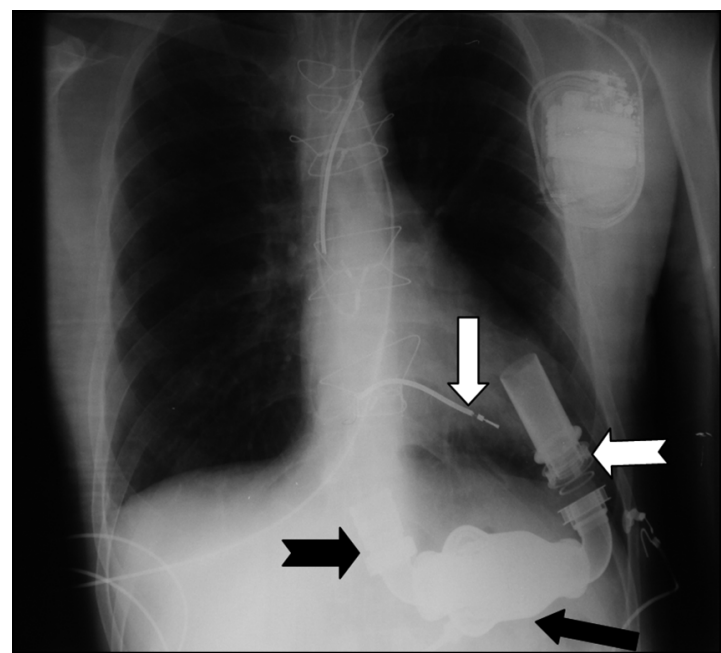

Figura 2. Radiografía de tórax. Se observa cánula de entrada en el ventrículo izquierdo (flecha con muesca blanca), cánula de salida hacia la aorta (flecha con muesca negra), bomba de flujo axial rotatorio que impulsa la sangre de forma continua (flecha negra) y cable del DAI (flecha blanca).

(Radiografía tórax, Figura 2). Al día siguiente se produjo donación. En pabellón se efectuó canulación femoral derecha para conexión a circulación extracorpórea. La extracción del HM II fue muy compleja por la cirugía previa y las múltiples adherencias, lo que dificultó el explante. Se realizó TxC ortotópico con técnica bicava. Se utilizó inducción de inmunosupresión con basiliximab (Simulect ${ }^{\circledR}$ ); triterapia con ciclosporina (Transporina ${ }^{\circledR}$, Recalcine, Chile) micofenolato mofetil (Linfonex ${ }^{\circledR}$, Recalcine, Chile), prednisona y profilaxis antibiótica con vancomicina. La evolución postoperatoria inicial fue favorable. Recibió apoyo vasoactivo con milrinona, adrenalina e isoproterenol. Se logró retiro de VM y extubación a las 12 $\mathrm{h}$ sin incidentes.

Al día siguiente del $\mathrm{TxC}$ evolucionó con trombosis venosa profunda ileofemoral derecha extensa en relación al sitio de canulación y flegmasia alba dolens. Desarrolló rabdomiolisis, con niveles de creatinkinasa total sobre $30.000 \mathrm{UI} / \mathrm{L}$, insuficiencia renal aguda oligoanúrica y en forma paralela shock severo, con patrón hemodinámico predominantemente distributivo, con altos requerimientos de drogas vasoactivas (DVA): milrinona, isoproterenol, adrenalina, vasopresina y noradrenalina. Se reintubó y conectó a VM y se inició terapia de soporte renal con hemodiafiltración veno - venosa continua diaria. Se amplió cobertura antibiótica con imipenem por intensa respuesta inflamatoria. La ecocardiografia de control mostró un VD dilatado e hipocinético, FEVI estimada en 59\% y un derrame pericárdico leve. Luego de 4 días de extrema gravedad se logró destetar de DVA, retirar de VM y se pasó a hemodiálisis intermitente.

Con posterioridad presentó deterioro gasométrico y alzas febriles. Se realizó un scanner torácico que demostró extensa ocupación pleural izquierda, reingresando a pabellón para la evacuación de un hemotórax. Se realizó una fibrobroncoscopía con toma de cultivos, aislándose Enterobacter. Completó 21 días de tratamiento antibiótico con imipenem con respuesta adecuada. Evolucionó con progresiva mejoría de condición general, recuperación de la función renal, suspendiéndose la diálisis luego de 20 días. La ecocardiografía de control mostró recuperación de la función del VD manteniendo una FEVI normal.

Inició rehabilitación intrahospitalaria, egresando luego de 58 días en buenas condiciones generales. Actualmente, se mantiene en controles periódicos en INT, en clase funcional I, con adecuada función del injerto, sin rechazos significativos tras 24 meses de trasplante cardiaco.

\section{Discusión}

Los VAD reemplazan la función del VI, en forma transitoria o definitiva. Pueden ser agrupados según su localización como paracorpóreos o intracorpóreos; según el tipo de flujo, en pulsátil o continuo; según se asista uno o los dos ventrículos en uni o biventriculares y según el tiempo de la asistencia en corta, mediana o larga duración ${ }^{8,9}$. Estos últimos pueden ser utilizados por meses o incluso años. A este grupo pertenece el HM II.

El estudio REMATCH demostró mejoría en la sobrevida con uso de VADs de flujo pulsátil, en comparación con terapia médica óptima en pacientes con IC CF IV no candidatos a trasplante ( $52 \%$ vs $25 \%$ al año de seguimiento $)^{10}$, sin embargo, la evidencia actual sugiere una mejor sobrevida con el uso de dispositivos de flujo continuo, como HM II ${ }^{\circledR}$, en comparación con los de flujo pulsátil en el seguimiento a largo plazo. (58 a $68 \%$ a 1 año y 24 a $55 \%$ a 2 años) ${ }^{11}$.

Según el registro INTERMACS (Interagency 
Registry Mechanically Assisted Circulatory Support) en 6 años se han implantado 6.000 dispositivos ${ }^{14}$. En el año 2011 se realizaron 1.500 implantes (5 veces más que el año 2006). Este aumento tiene que ver con la aprobación por parte de la FDA (Food and Drug Administration) de los Estados Unidos de Norteamérica de VADs para uso prolongado como puente a trasplante y terapia de destinación a partir del año $2008^{14}$.

HM II fue uno de los primeros dispositivos implantables y de larga duración en ser aprobado y una de sus ventajas es que funciona a través de un mecanismo electromagnético que genera una acción rotatoria, lo que permite un flujo continuo de la sangre, con mínimo roce, disminuyendo la formación de trombos. Es más pequeño, liviano y fácil de portar. Ha mostrado una menor frecuencia de arritmias cardiacas, infecciones, falla renal y respiratoria, pero además reduce la necesidad de rehospitalizaciones hasta en $36 \%{ }^{15}$.

En relación a sus resultados quirúrgicos, con HM II existe un mayor número de implantes exitosos y menor necesidad de reoperaciones. Si bien el explante es difícil, hay comunicaciones de explantes exitosos incluso con cirugía mínimamente invasiva ${ }^{16}$.

\section{Conclusiones}

Los VADs representan una alternativa válida y efectiva para pacientes con IC avanzada, como puente a trasplante para mejorar la condición general del paciente y la función de órganos y sistemas, permitiendo además el egreso hospitalario, como en el caso presentado, pero también como terapia de destinación en pacientes con contraindicaciones para efectuar un TxC. Los dispositivos de flujo continuo para uso prolongado, como HM II, ofrecen un beneficio superior en términos de sobrevida comparado con los dispositivos de flujo pulsátil. En Chile no existe experiencia con estos dispositivos por el elevado costo, además porque el número de receptores para TxC aún es bajo y los tiempos de espera son más cortos (21 días promedio para status IA y 100 días para status II en INT). Sin embargo, podría representar una opción para aquéllos pacientes que se consideren no tributarios de un TxC y que reúnen ciertas condiciones tales como ausencia de enfermedades neoplásicas o fallas orgánicas avanzadas que contraindiquen incluso el implante del dispositivo.

\section{Referencias}

1. Ho KK, Pinsky JL, Kannel WB, Levy D. Epidemiology of heart failure: the Framingham Study. J Am Coll Cardiol 1993; 22 (Suppl A): 6A-13A.

2. Massie BM. Shah NB. Evolving trends in the epidemiologic factors of heart failure: Rationale for preventive strategies and comprehensive disease management. Am Heart J 1997; 133: 703-12.

3. Roger V, Go A, Lloyd-Jones D, Adams R, Berry J. Brown T, et al. Heart disease and stroke statistics: 2011 update. A report from American heart Association. Circulation. 2011; 123: e18-e209.

4. Bristow MR. Saxon L, Boehmer J, Krueger S, Kass D, De Marco T, et al. Cardiac-Resynchronization Therapy with or without an Implantable Defibrillator in Advanced Chronic Heart Failure. N Engl J Med 2004; 350: 2140-50.

5. Costanzo MR, Mills RM, Wynne J. Characteristics of "Stage D" heart failure: Insights from the Acute Decompensated Heart Failure National Registry Longitudinal Module (ADHERE LM). Am Heart J 2008; 155 (2): 339-47.

6. Stehlik J, Edwards L, Kuchergavayan A, Benden C, Christie JD, Dobbels F, et al. The Registry of the International Society for Heart and Lung Transplantation: 29th Official Adult Heart Transplant Report-2012. J Heart Lung Transplant 2012; 31 (10): 1045-95.

7. Barr ML, Bourge RC, Orens JB, Mc Curry K, Rivy WS, Hulbert-Shawn TE, et al. Thoracic organ transplantation in the United States, 1994-2003. Am J Transplant 2005; 5: 934-49.

8. Peura J, Colvin-Adams M, Francis G, Grady K, Hoffman $\mathrm{T}$, Jessup $\mathrm{M}$, et al. Recommendations for the Use of Mechanical Circulatory Support: Device Strategies and Patient Selection. Circulation 2012; 126: 2648-67.

9. Kirklin JK, Naftel D. Mechanical Circulatory Support: Registering a Therapy in Evolution. Circ Heart Fail 2008; 1: 200-5.

10. Rose E, Gelijns A, Moskowitz A, Heitjan D, Stevenson L, Dembitsky W, et al. Long-Term Use of a Left Ventricular Assist Device for End-Stage Heart Failure. N Engl J Med 2001; 345: 1435-43.

11. Slaughter M, Rogers J, Milano C, Russell S, Conte J, Feldman D, et al. Advanced Heart Failure Treated with Continuous-Flow Left Ventricular Assist Device, N Engl J Med 2009; 361: 2241-51.

12. Villavicencio M, Turner R, Larrea R, Larraín E, Turner F, Rivera J, et al. Puente al trasplante de 4 semanas utilizando el sistema de asistencia ventricular Levitronix Centrimag ${ }^{\circledR}$ en el shock cardiogénico post-infarto al miocardio. Rev Med Chile 2010; 138: 752-7.

13. Castro P, Baraona F, Baeza C, Mac-nab P, Berlina A, 
Primer puente a trasplante cardiaco exitoso en Chile usando el dispositivo Heart Mate II - N. Bunster et al

Zalaquett $\mathrm{R}$, et al. Ventricular assist device as a bridge to transplant in patients with cardiogenic shock. Preliminary experience in Chile with Abiomed BVS 5000. Rev Med Chile 2006; 134 (8): 1019-23.

14. Kirklin JK, Naftel DC, Kormos RL, Stevenson LW, Pagani FD, Miller MA, et al. Fifth INTERMACS annual report: Risk factor analysis from more than 6,000 mechanical circulatory support patients. J Heart Lung Transplantation 2013; 32: 141-56.
15. 32:141-156.ion 213; 1 report: Risk factor analysis from more than 6,000 mechanical circulatory support patients. J. Heart Lung 15. Advanced Practice Guidelines for Heart Mate Destination Therapy. Pleasonton, Calif: Thoratec Corporation; 2004.

16. Cheung A, Bashir J, Kaan A, Kealy J, Moss R, Shayan H. Minimally invasive, off-pump explant of a continuousflow left ventricular assist device. J Heart Lung Transplant 2010; 29 (7): 808-10. 